tissues of $\mathrm{Becn}^{+/-}$mice where spontaneous tumours develop. The authors suggest that haploinsufficiency of Becn1 is involved in tumour suppression and that decreased autophagy might contribute to increased cell growth, so promoting tumorigenesis.

So, BECN1 is the first downstream autophagy-execution gene to be linked to human cancer. Genes best known for inducing cell growth or inhibiting apoptosis — such as phosphatidylinositol 3-kinase, $A K T$ and PTEN - are also involved in regulating autophagy, and the observations made in this study with $B E C N 1$ indicate that regulation of autophagy might contribute to their roles in oncogenesis too.

Ezzie Hutchinson

(0) References and links ORIGINAL RESEARCH PAPER $Q u$, $X$. et al. Promotion of tumorigenesis by heterozygous disruption of the beclin 1 autophagy gene. J. Clin. Invest. 112, 1809-1820 (2003)

\section{WEB SITE}

Beth Levine's lab:

http://cpmcnet.columbia.edu/dept/id/beth_levine ,_md.htm

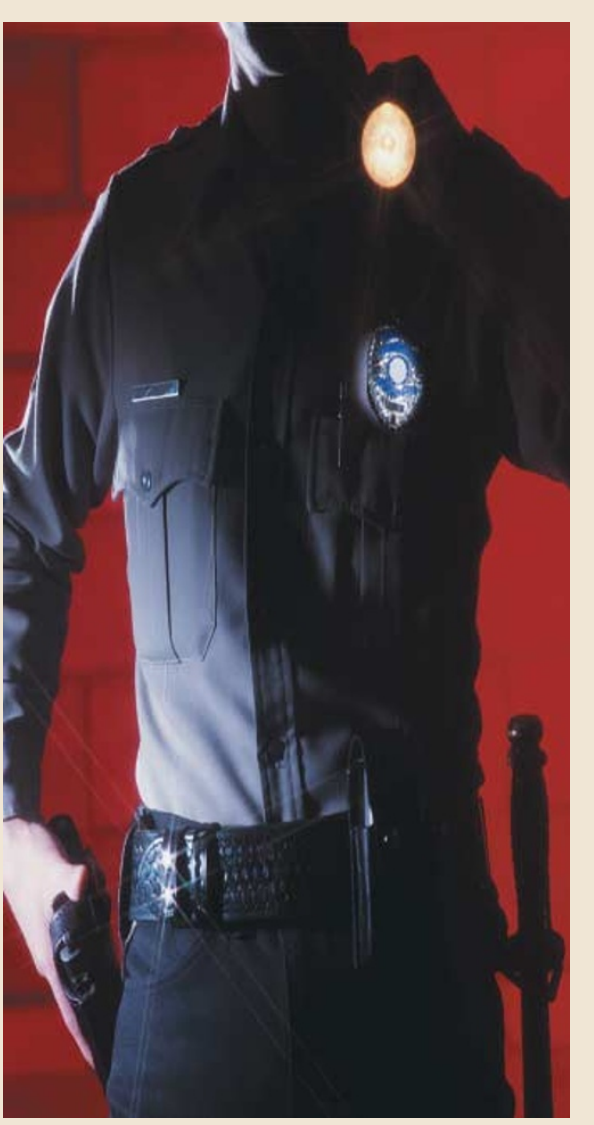

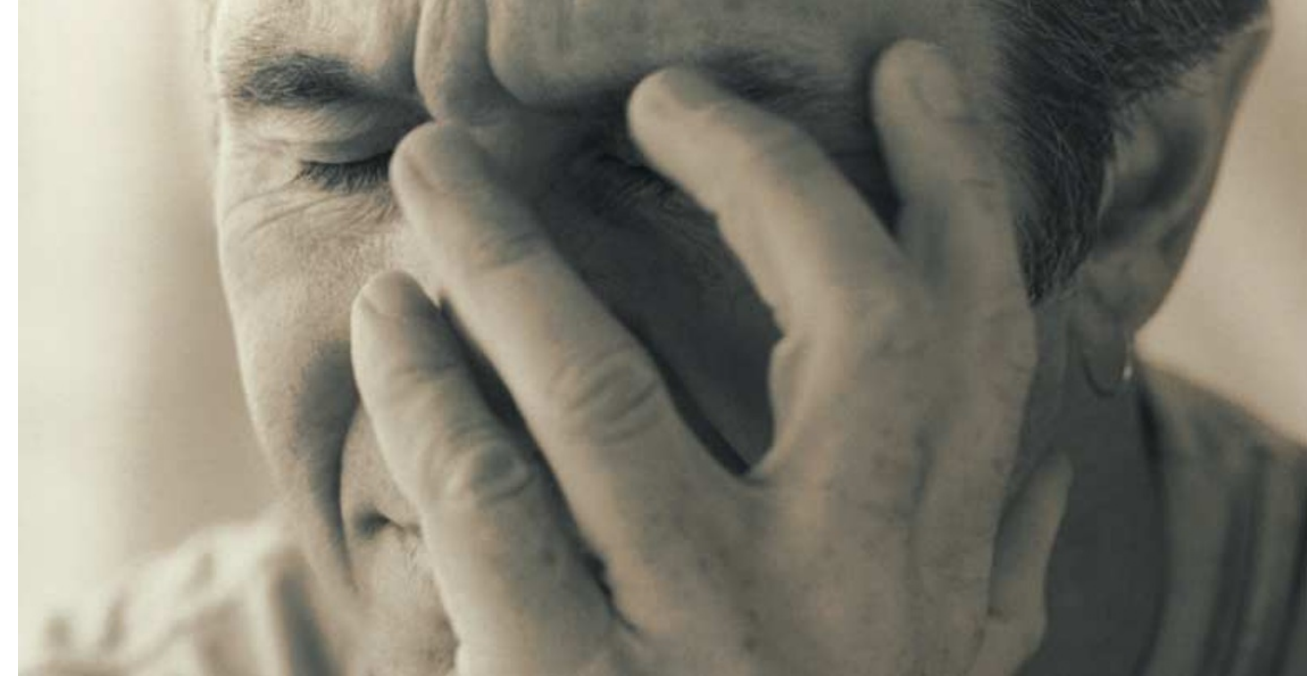

TUMOUR SUPPRESSORS

\title{
BRCA2: preventing a breakdown
}

Mutations in the tumour-suppressor gene BRCA2 cause genomic instability and predisposition to cancer. However, the exact function of BRCA2 in safeguarding genomic integrity in normal cells is unclear. New evidence from Ashok

Venkitaraman's group indicates that BRCA2 is required to prevent the breakdown of stalled replication forks and that disruption of this function leads to the chromosomal rearrangements that occur spontaneously in dividing BRCA2-mutant cells.

Although it is not part of the normal replication machinery, BRCA2 localizes in the nuclear foci that are formed when replication is inhibited. So, does BRCA2 function in the cellular response to stalled replication? Venkitaraman and colleagues investigated this by blocking DNA synthesis in mouse cells that were homozygous for a targeted truncation of $\mathrm{BrCa} 2-\mathrm{BrCa}^{\mathrm{Tr}}$ - that produces a nonfunctional protein. They used two-dimensional gel electrophoresis to visualize Y-arcs - DNA structures that are formed at replication forks - at the ribosomal DNA (rDNA) locus. When replication was inhibited using hydroxyurea (HU), these structures disappeared from $\mathrm{Brca}^{\mathrm{Tr} / \mathrm{Tr}}$ cells, but persisted in wild-type cells, indicating that $\mathrm{Brca} 2$ is required for the stability of stalled replication forks.

In replication-defective bacteria, stalled replication forks can be broken down into linear chromosomal fragments. If a similar process occurred as a result of loss of $\mathrm{BrCa} 2$ function, this could explain the spontaneous genomic instability that arises as a result of mutations in this gene. To investigate this, the authors used pulsed-field gel electrophoresis (PFGE) to check for the production of DNA fragments that would be predicted to form because of the breakdown of replication forks in the rDNA locus.

Chromosomal DNA from this locus is usually too large to be visualized by PFGE, but in $\mathrm{Brca} 2^{\mathrm{Tr} / \mathrm{Tr}}$ cells, fragments consistent with breakdown at rDNA replication forks were seen following application of HU. This effect of Brca2 inactivation is not restricted to the rDNA genes, as similar breakdown events were shown to occur at other genomic loci.

Importantly, some DNA breakage was also seen in $\mathrm{BrCa}^{\mathrm{Tr} / \mathrm{Tr}}$ cells that were not treated with HU. This indicates that loss of Brca2 function has a similar effect on the stability of replication forks that stall during normal replication - because of DNA lesions or at natural pause sites - as it does in response to a global arrest of replication.

In response to arrested DNA replication, mammalian cells activate a Chk2-dependent checkpoint mechanism that prevents cells from prematurely undergoing mitosis. Does the role of Brca2 in response to replication arrest lie in the activation of this checkpoint? Venkitaraman and colleagues found that $\mathrm{BrCa}^{\mathrm{Tr} / \mathrm{Tr}}$ cells did not enter mitosis prematurely after treatment with $\mathrm{HU}$, and showed that the activated, phosphorylated form of Chk2 is present in these cells. So, Brca2 functions either downstream of Chk2 or as part of an independent pathway.

The breakdown of replication forks as a result of Brca2 inactivation provides the first example of a link between human disease and defects in the response to arrested DNA replication. It will be interesting to see whether similar mechanisms underlie other conditions in which chromosomal instability is related to cancer predisposition.

Louisa Flintoft

\section{(2) References and links}

ORIGINAL RESEARCH PAPER Lomonosov, M., Anand, S., Sangrithi, M. Davies, R. \& Venkitaraman, A. R. Stabilization of stalled DNA replication forks by the BRCA2 breast cancer susceptibility protein. Genes Dev. 17, 3017-3022 (2003)

WEB SITE

Ashok Venkitaraman's lab: http://www.hutchison-

mrc.cam.ac.ukNenkitaraman.html 